\title{
Future projections of extreme precipitation using Advanced Weather Generator (AWE-GEN) over Peninsular Malaysia
}

\author{
A. H. SYAFRINA ${ }^{1}$, M. D. ZALINA ${ }^{1}$ \& L. JUNENG ${ }^{2}$ \\ 1 UTM Razak School of Engineering and Advanced Technology, Universiti Teknologi Malaysia, Jalan Semarak, \\ 54100 Kuala Lumpur, Malaysia \\ syafrinahalim@gmail.com \\ 2 School of Environment Natural Resources, Faculty of Science and Technology, Universiti Kebangsaan Malaysia, \\ 43600 UKM Bangi, Selangor, Malaysia
}

\begin{abstract}
A stochastic downscaling methodology known as the Advanced Weather Generator, AWE-GEN, has been tested at four stations in Peninsular Malaysia using observations available from 1975 to 2005 . The methodology involves a stochastic downscaling procedure based on a Bayesian approach. Climate statistics from a multi-model ensemble of General Circulation Model (GCM) outputs were calculated and factors of change were derived to produce the probability distribution functions (PDF). New parameters were obtained to project future climate time series. A multi-model ensemble was used in this study. The projections of extreme precipitation were based on the RCP 6.0 scenario (2081-2100). The model was able to simulate both hourly and 24-h extreme precipitation, as well as wet spell durations quite well for almost all regions. However, the performance of GCM models varies significantly in all regions showing high variability of monthly precipitation for both observed and future periods. The extreme precipitation for both hourly and 24-h seems to increase in future, while extreme of wet spells remain unchanged, up to the return periods of $10-40$ years.
\end{abstract}

Key words stochastic downscaling, General Circulation Model; multi-model; weather generator; extreme precipitation

\section{INTRODUCTION}

Climate change is a global issue which represents an important challenge for our society as it obliges us to adapt our actions to a not well-known future climate. Change in rainfall is one of the most critical impacts of climate change, which brings changes in the probabilities and risks of flooding (Willem et al. 2011). Furthermore, the possible changes of intensity and frequency of extreme rainfall are also increasing due to the enhanced greenhouse effect (Huang et al. 2011). These phenomena have attracted many meteorologists and hydrologists in the world to investigate the spatial and temporal characteristics of precipitation extremes. It is believed that both frequency and intensity of extreme precipitation events are the major impacts of global warming (Sen Roy, 2009, Cheng et al. 2012). The General Circulation Model (GCM) can reproduce important processes about global- and continental scale atmosphere and predict future climate under different emission scenarios. Although there are many uncertainties in different GCMs, they are still the most adapted approach, to date, for obtaining information on climate change (Chu et al. 2010).

Unfortunately, GCMs are usually at resolution that is too coarse (generally greater than $2.0^{\circ}$ for both latitude and longitude, and greater than $200 \mathrm{~km}$ for middle latitudes) for many climate change impact studies (Fatichi et al. 2011, Hashmi et al. 2011). Additionally, the internal relationships between the model's variables produced from GCMs may not always be the same as those found in the observational data. As a result, their simulations of current regional climate can often be inaccurate for sub-grid scales. The discrepancy between the scale at which the models deliver output and the scale that is required for most impact studies has led to the development of downscaling methodologies. Two general approaches are the dynamical and statistical downscaling. Statistical downscaling can be essentially divided into three categories: regression methods, weather pattern-based approaches, and stochastic weather generators. In this study we investigate the capability of a stochastic downscaling method, the Advanced Weather Generator (AWE-GEN), in projecting future climate variables in peninsular Malaysia.

\section{DATA}

Peninsular Malaysia is located between $1^{\circ} \mathrm{N}$ and $6^{\circ} \mathrm{N}$ in the northern hemisphere and between $100^{\circ} \mathrm{E}$ to $103^{\circ} \mathrm{E}$ longitude, which experiences differences in rainfall across all regions. Four 
stations representing the north, south, west, and east of Peninsular Malaysia (Fig. 1) were used in the study, with observations from 1975 to 2005 . This period represents the interval of the control scenario, for which both observations and climate model simulations were used. The future climate scenario is based on GCM projections for the period 2081-2100. Hourly rainfall, temperature, wind speed, and relative humidity data were obtained from the Malaysian Drainage and Irrigation Department (DID) and Malaysian Meteorological Department (MMD). GCMs realizations were obtained from the dataset compiled in the World Climate Research Programme's (WCRP's), Coupled Model Intercomparison Project phase 5 (CMIP5). To take uncertainties into account, an ensemble of multi-model were used in this study such as GFDL-CM3 (United States), IPSLCM5A-LR (Paris, France), MIROC5 (Japan), MRI-CGCM3 (Japan) and NorESM1-M (Norway).

\section{METHOD}

The AWE-GEN model is made up of several components. The following sections will give a brief description of the components.

\section{Neyman-Scott Rectangular Pulses (NSRP)}

In the NSRP model, the arrival times of storm origins occur in a Poisson process with rate $\lambda$ where each storm origin generates a random number $C$ of cell origins. The rectangular pulse $(L, X)$ is associated with each cell origin, where $L$ and $X$ are independent random variables corresponding to the lifetime and intensity of the pulse, respectively. The pulse represents a rain cell where $X_{t-u}(u)$ is an independent random variable representing the rainfall intensity at time $t$ due to a cell with starting time $t-u$, and $\delta N(t) \equiv N(t, t+\delta)$ is the number of cell origins in the time interval $(t, t+\delta)$. The total intensity at time $t, Y(t)$ is the summation of the intensities of all cells alive at time $t$ where $L$ is exponentially distributed with mean $\eta$. The waiting time for a cell origin after a storm origin is independently exponentially distributed with mean $\beta$ where no cell origin occurs at the storm origin. $C$ is taken to be a geometric random variable with mean $\mu_{c}$. The gamma distribution with parameters $(\alpha, \theta)$ is selected for the random variable $X$. At least six equations are needed to estimate these parameters.

The objective function containing statistical properties of rainfall at different aggregation times is used. The properties used in the objective function are the coefficient of variation $C_{h}(h)=\sqrt{\gamma_{h, 0}} / \mu_{h}$, the lag-1 auto-correlation $\rho(h)=\gamma_{h, 1} / \gamma_{h, 0}$, the skewness $\kappa(h)=\xi_{h} / \gamma_{h, 0}^{3 / 2}$ and the probability that an arbitrary interval of length $h$ is dry, $\Phi(h)$. The parameters $\mu_{h}, \gamma_{h, l}$ and $\xi_{h}$ represent the mean, the covariance, and the third moment of precipitation process at a given aggregation time interval $h$ and lag $l$. The procedure specifically uses the above statistical properties of the precipitation process at four different time scales $h: 1,6,24$, and $72 h$. The simplex method is used as a minimization method for the imposed objective function. In order to take into account the seasonality of site climatology, the parameters are estimated on a monthly basis meaning six parameters $\left(\lambda, \mu_{c}, \beta, \eta, \alpha\right.$ and $\left.\theta\right)$ for each month need to be inferred to completely define the NSRP model. Rodriguez-Iturbe et al. (1987a) gives a full account of the NSRP methodology.

\section{Autoregressive Lag-1 (AR1) model}

The AR1 model is employed to preserve the variance and the autocorrelation properties of the precipitation process at the annual scale. The AR1 model is:

$$
P_{y r}(i)=\bar{P}_{y r}+\rho_{P_{y r}}\left(P_{y r}-\bar{P}_{y r}\right)+\eta(i) \sigma_{P_{y r}} \sqrt{1-\rho^{2} P_{y r}}
$$


where $\bar{P}_{y r}[\mathrm{~mm}]$ is the average annual rainfall, $\sigma_{P_{y r}}$ is the standard deviation and $\rho_{P_{y r}}$ is the lag-1 autocorrelation of the process. The term $\eta(i)$ represents the random deviate of the process which is transformed according to the Wilson-Hilferty approach. The parameters $\bar{P}_{y r}, \sigma_{P_{y r}}, \rho_{P_{y r}}$ and $\gamma_{P_{y r}}$ are determined from the annual observations.

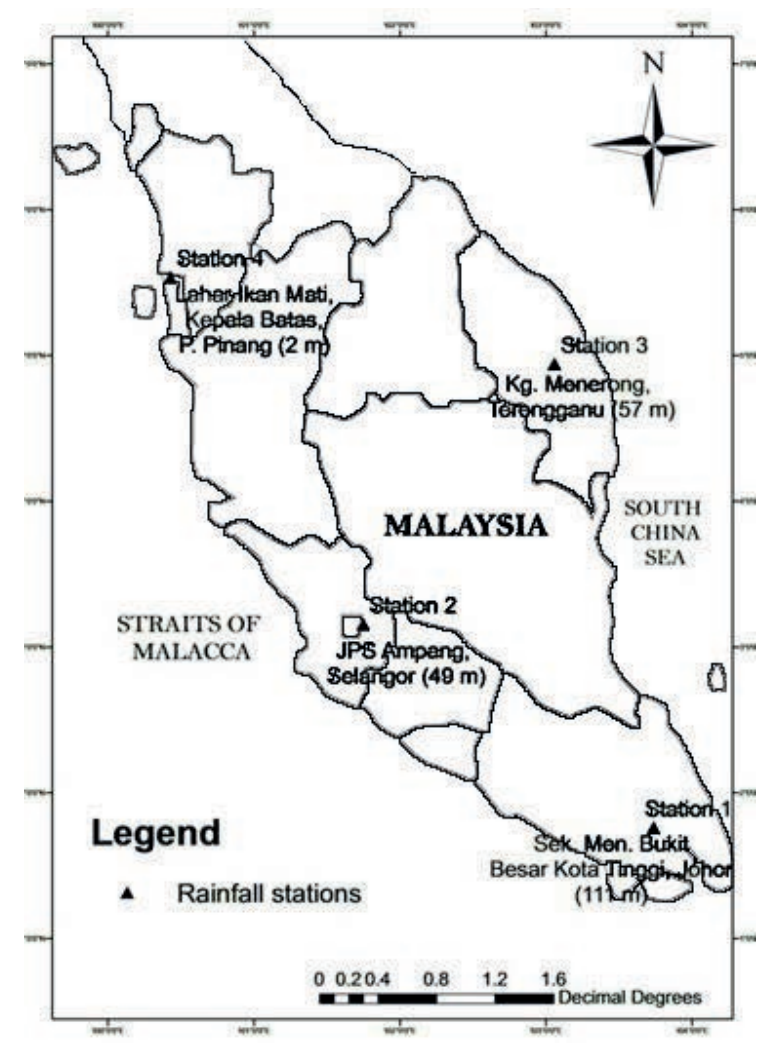

Fig. 1 Location of the four stations used in this study. The values in bracket represent the elevation of the station.

\section{Factor of change}

In AWE-GEN, the factor of change (FC) provides projections for the change between the present climate and the future climate. These FCs are then used to perturb the statistically derived time series to generate statistical expressions of hourly future time series. The climate statistical properties for a given area are downscaled from GCMs realizations. The statistical properties considered are: the mean $E_{\mathrm{Pr}}(h)$, the variance $V A R_{\mathrm{Pr}}(h)$ and the skewness $S K E_{\mathrm{Pr}}(h)$ where $h$ is the aggregation interval. Each of these properties is calculated on a monthly basis to include the effects of intra-annual seasonality. The factor of change for precipitation is:

$$
S(h)^{F U T}=\left[\frac{S(h)^{G C M, F U T}}{S(h)^{G C M, C T S}}\right] S(h)^{O B S}
$$

and, for temperature is:

$$
T_{m o n}^{F U T}=T_{m o n}^{O B S}+T_{m o n}^{G C M, F U T}-T_{m o n}^{G C M, C T S}
$$

where $S(h)$ denotes the statistical property at $h$ aggregation interval, FUT denotes the future scenario, OBS denotes observations, and CTS denotes the control scenario while the GCM denotes the climate model. 


\section{Multi-model ensemble approach}

A Bayesian statistical model is employed in this study. All uncertain quantities are modelled as random variables, with a priori probability distributions. The objective is to capture the posterior distributions of the product $(v / \mu)$ or additive $(v-\mu) \mathrm{FC}$ where $\mu$ and $v$ represent the true values of a certain variable for the control scenario and future climate, respectively. The method assigned weights to climate models, according to two criteria: the bias and convergence. The bias measures the difference between GCM simulations and the best approximation of the truth $\bar{\mu}$ while the convergence measures the distance between the GCM simulations and the "consensus" estimate $\bar{v}$ of future realizations. Each statistic $\bar{v}$ represents the expected value of the probability distribution functions (PDF) for the future.

\section{RESULTS AND DISCUSSION}

The performance of the model was first tested during the control period. Simulated results were compared to observed data within this period. Figure 2 shows the result for stations 1 and 2 (similar results were obtained for stations 3 and 4). There is a considerable overlap between the simulated and observed extreme precipitation, up to the return periods of 20-40 years. However, the hourly extreme precipitation is somewhat underestimated for both Station 1 and Station 3. In contrast, the hourly extreme precipitation for Station 2 and Station 4 is well simulated. Meanwhile, the 24-h aggregation period of extreme precipitations are well simulated at all stations. Extremes of wet spell durations are generally well captured by the model, but extreme dry spells are somewhat underestimated for all stations. Dry/wet spell duration is the number of consecutive days with precipitation depth lower/larger than $1(\mathrm{~mm})$. Figure 3 shows the factors of change for air temperature and precipitation for the month of November for stations 1 and 3 (again similar results were found for the other months and stations).

Station 1
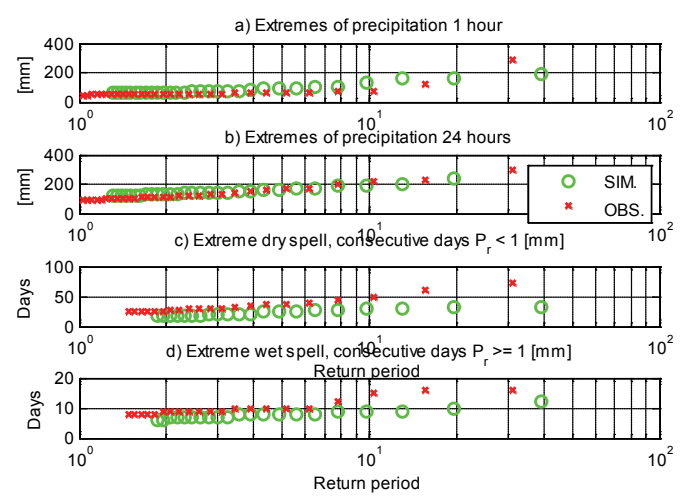

Station 2

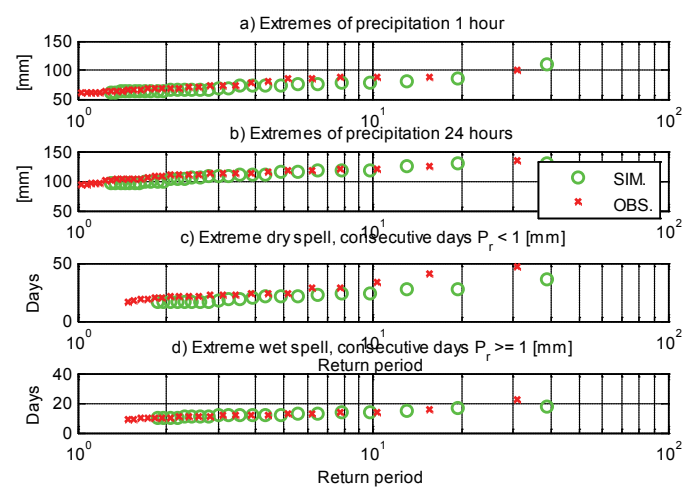

Fig. 2 A comparison between the observed and simulated values of extreme precipitation at (a) 1-h and (b) 24-h aggregation periods; (c) extremes of dry and (d) wet spell durations.

Future projections of monthly and extreme precipitation using the new set of AWE-GEN parameters derived from the mean factors of change and downscaled to finer time scales are the final outputs of the study. Overall, the mean amount of monthly rainfall is expected to be higher throughout all four stations with high variability of future monthly rainfall amount at Stations 1 and 2, but less variability at Station 3 (Fig. 4). Finally, the comparison between the observed and future values of extreme precipitations of hourly and 24-h aggregation periods is revealed in Fig. 5. It is shown that the extreme precipitation for both aggregation periods seems to increase in future. In particular, the future hourly extreme for Station 1 and Station 3 are expected to increase during the return period of 10-40 years with precipitation exceeding $200 \mathrm{~mm}$ and $100 \mathrm{~mm}$, respectively.

Meanwhile, the future hourly extreme for Station 2 and Station 4 are expected to increase during the return period of 10-40 years with $100 \mathrm{~mm}$ maximum precipitation. As for the future 

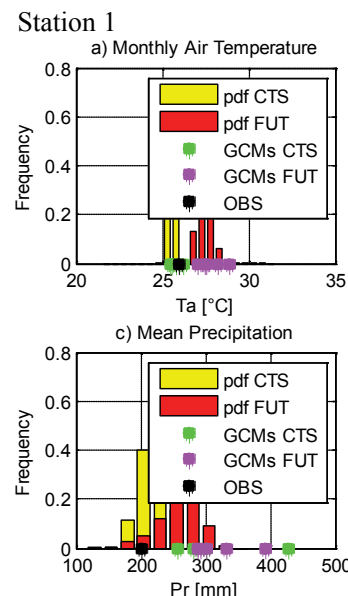
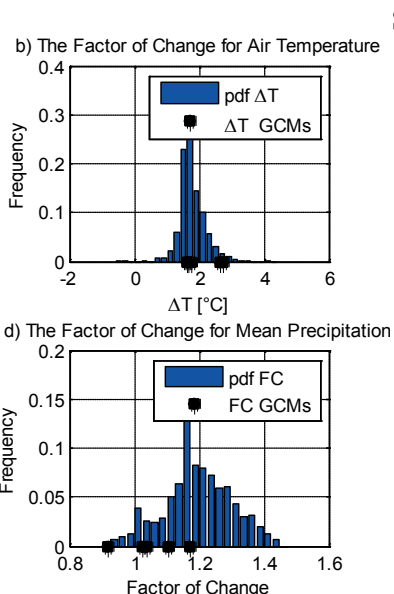

Station 2

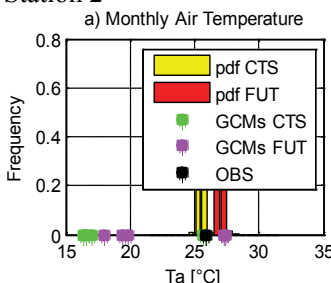

c) Mean Precipitation

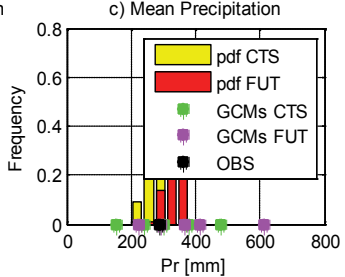

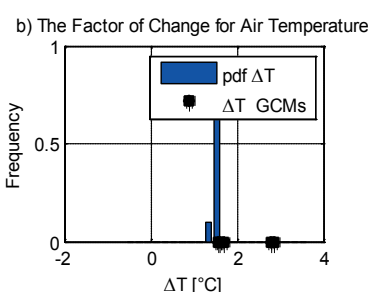

d) The Factor of Change for Mean Precipitatio

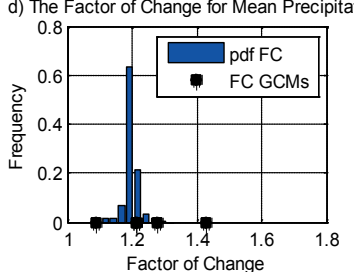

Fig. 3 The posterior probability density functions (PDF) of mean temperature and precipitation obtained from the multi-model ensemble for all stations, for November.

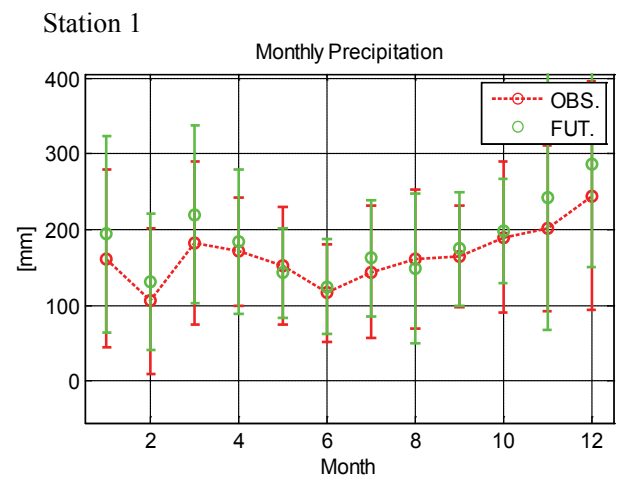

surians

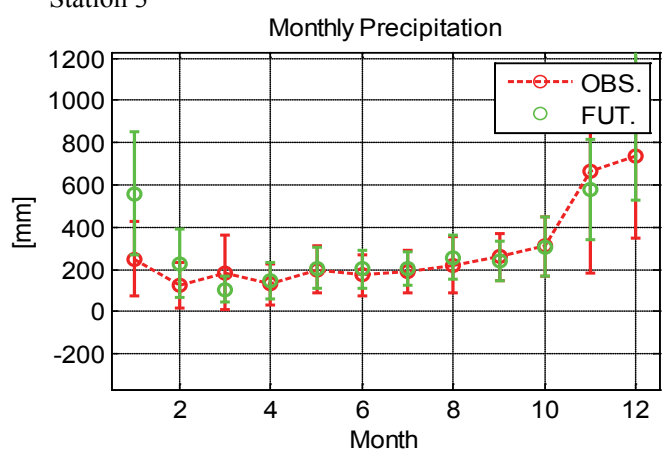

Station 2

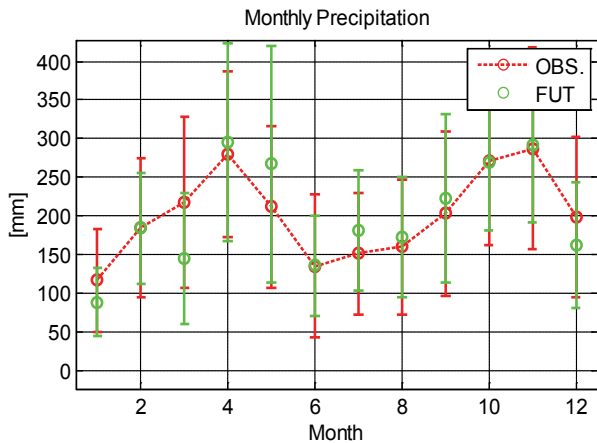

Station 4

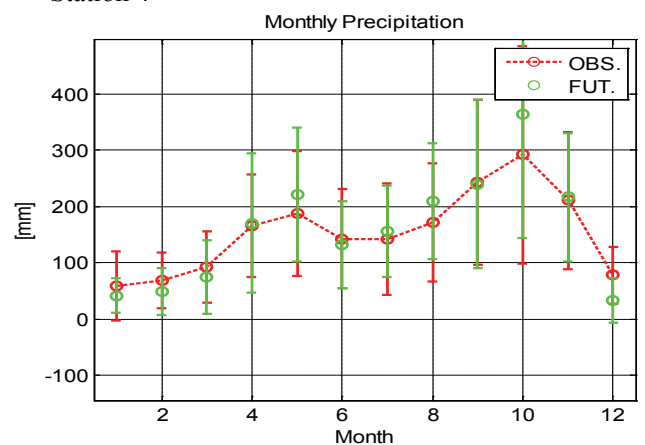

Fig. 4 A comparison between observed ('--o') and future ('o') monthly precipitation. The vertical bars denote the standard deviations of the monthly values.

24-h aggregation periods of extreme precipitation, Station 1 and Station 4 are expected to increase during the return period of 10-40 years with precipitation exceeding $450 \mathrm{~mm}$. Nevertheless, the future 24-h extreme for Station 2 and Station 3 are expected to increase during the return period of 10-40 years with precipitation exceeding $200 \mathrm{~mm}$ and $1000 \mathrm{~mm}$, respectively. An extreme dry spell for all stations is projected to decrease in future, while the extreme wet spell for all stations will remain unchanged in the future, up to the return periods of 10-40 years.

\section{CONCLUSION}

Generally, the average in total monthly rainfall will increase in future, but with a high variability. However, there is slight difference with the variability of the total monthly rainfall projections at stations located on the east coast showing lower variability. Overall, the scenario of the future 
extreme precipitation for both hourly and 24-h in Peninsular Malaysia is expected to increase, up to the return periods of $10-40$ years.

Station 1
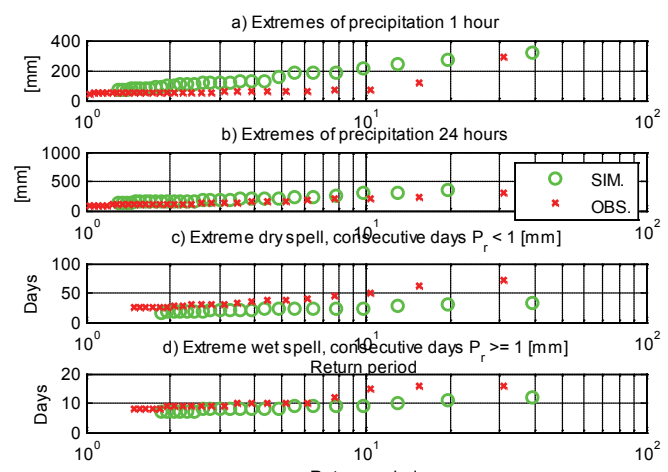

Return period

Station 3
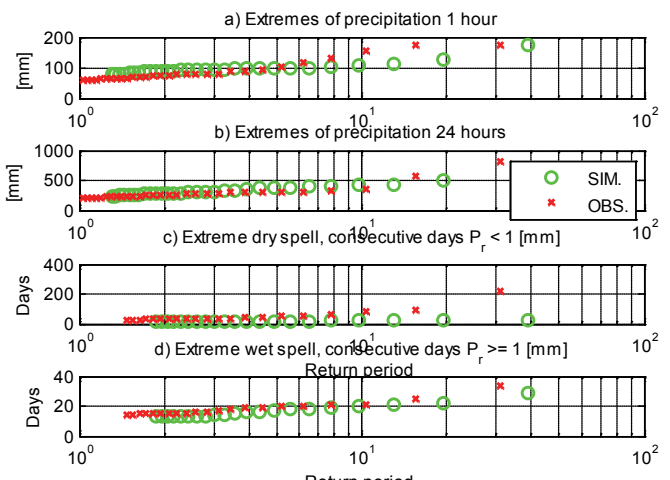

Station 2
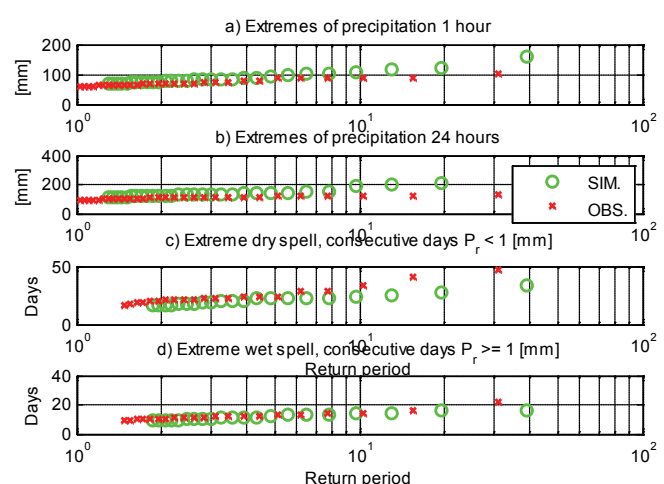

Station 4

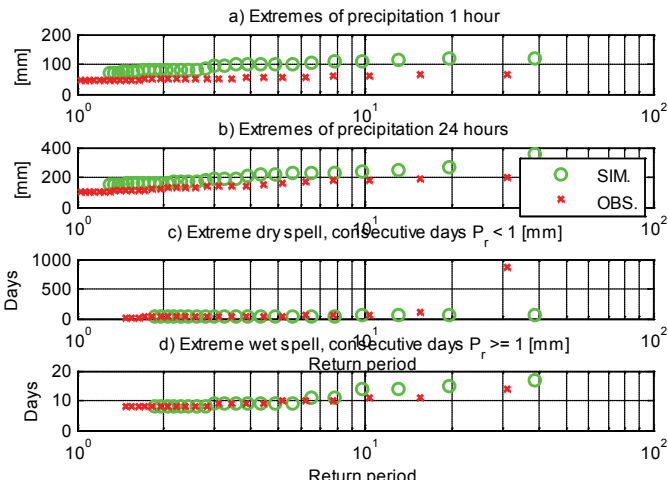

Fig. 5 A comparison between the observed and future values of extreme precipitations at (a) 1-h and (b) 24-h aggregation periods; (c) extremes of dry and (d) wet spell durations. Dry/wet spell duration is the number of consecutive days with precipitation depth lower/larger than $1(\mathrm{~mm})$.

Acknowledgements This work was supported by the Malaysian Drainage and Irrigation Department (DID), Malaysian Meteorological Department (MMD) and World Climate Research Programme's (WCRP's), Coupled Model Intercomparison Project phase 5 (CMIP5) in providing us the observed and future climate data. This research was funded by Universiti Teknologi Malaysia research vote GUP: 09J19. We would also like to thank Fatichi et al. (2011) for making available the downscaling file program for this research to be done.

\section{REFERENCES}

Cheng, C. S., et al. (2012) Possible impacts of climate change on extreme weather events at local scale in south-central Canada. Climatic Change 112, 963-979.

Chu, P. S., et al. (2010) Extreme rainfall events in the Hawaiian islands. J. Appl. Met. Climatol. 48(3), 502-516.

Fatichi, S., Ivanov, V. Y., and Caporali, E. (2011) Simulation of future climate scenarios with a weather generator. Adv. Water Resour. 34(4), 448-467.

Hashmi, M. Z., Shamseldin, A. Y. and Melville, B. W. (2011) Comparison of SDSM and LARS WG for simulation and downscaling of extreme precipitation events in a watershed. Stoch. Env. Res. Risk A. 25(4), 475-484.

Huang, C. Y., Wong, C. S. and Yeh, T. C. (2011). Extreme rainfall mechanisms exhibited by Typhoon Morakot (2009). Terr. Atmos. Ocean Sci. 22, 613-632.

Rodriguez-Iturbe, I and Eagleson, P. S. (1987a). Mathematical models of rainstorm events in space and time. Water Resour. Res. 23(1), 181-190.

Sen Roy, S. (2009) A spatial analysis of extreme hourly precipitation patterns in India. Int. J.Climatol. 29, 345-355.

Willems, P., et al. (2011) Climate change impact assessment on urban rainfall extremes and urban drainage: Methods and shortcomings. Atmos. Res. 103, 106-118. 